\title{
Structure Nanoclusters with a Well Defined Number of Atoms Using
}

\section{Aberration-Corrected STEM}

\author{
M. Jose-Yacaman
}

Department of Physics and Astronomy, University of Texas at San Antonio, One UTSA Circle, San

Antonio, TX , 78249

The determination of structure of molecular nanocrystal by X-ray diffraction has been achieved only in few cases as it requires homogenous, high degree of ordering, single crystal of oriented molecules[1, 2]. We show an alternative technique that can be used in determination of molecular crystal structures of nanoclusters having well defined number of atoms $\left(\mathrm{Au}_{144}\right.$ and $\mathrm{Au}_{333}$ ) without the fabrication of single crystal. We fabricate nanoclusters by using modified Brust's method and use size selection techniques to separate the desired fraction .MALDI TOF mass spectrometry[3] was used to determine the mass of the nanoparticles We used a combination of low voltage scanning transmission electron microscopy (STEM) electron diffraction and high angle annular dark field (HAADF) STEM images in a Cs-corrected JEOLJEM-ARM STEM to determine the crystal structure. The electron diffraction patterns and HAADF-STEM images are compared with the theoretical simulations of images and diffraction patterns obtained from atomistic structural models derived through first-principles density functional theory (DFT) calculations.It should be noted that the cleanliness of the sample, operating voltage of the microscope and exposure time of beam to the sample plays significant role in order to get atomically resolved images without altering the particle shape and attached ligands. The structural model obtained for $\mathrm{Au}_{144}\left(\mathrm{SCH}_{3}\right)_{60}$ by using first-principles DFT optimization and viewed along 3-fold axis is presented in Fig. 1a where the Au atoms: 54 in inner core, 60 in outer shell and 30 ad-atoms are represented by orange, brown and yellow color respectively while 60 sulfur atoms are represented by red color. Figure $1 \mathrm{~b}$ and c represents the simulated HAADF STEM images along different orientations. The experimental HAADF STEM images obtained for $\mathrm{Au}_{144}$ and $\mathrm{Au}_{333}$ nanoclusters are presented in Fig. 2a-d and Fig. 2e-h respectively. The corresponding FFT pattern is inset in each case. Based on the number of reflections and angles from FFT pattern, the corresponding structure is simulated. The STEM electron diffraction of $\mathrm{Au}_{144}$ and $\mathrm{Au}_{333}$ nanocluster is presented in Fig. 3a and b respectively. The number of reflection points and angle between them is measured and compared with the simulated ones. The combination of HAADF-STEM images (experimental and simulated) with diffraction patterns (experimental and simulated) will be used to investigate the crystal structure with well-defined number of atoms. We will also report the findings for other clusters such as $\mathrm{Au}_{333}$ and $\mathrm{Au}_{130}$

\section{References:}

[1]Tran, N. T.; Powell, D. R.; Dahl, L. F. Angewandte Chemie International Edition 2000, 39, 4121.

[2]Whetten, R. L.; Price, R. C. Science 2007, 318, 407

[3]Brust, M.; Schiffrin, D. J.; Bethell, D.; Kiely, C. J., Advanced materials 1995, 7, 795[4]Acknowledgments: The authors would like to acknowledge NSF grants DMR-1103730, "Alloys at the Nanoscale: The Case of Nanoparticles Second Phase" and PREM: NSF PREM Grant \# DMR 0934218. 

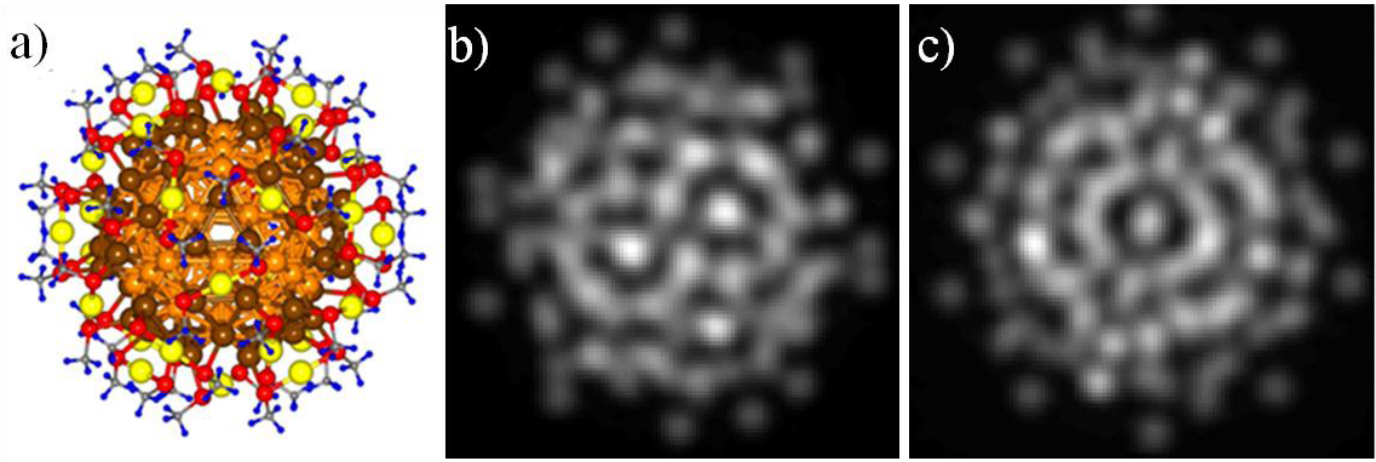

Fig. 1. a) Structural model of $\mathrm{Au}_{144}\left(\mathrm{SCH}_{3}\right)_{60}$ viewed along 3-fold axis obtained through firstprinciples DFT optimization. b) and c) Simulated HAADF STEM images along different orientations.

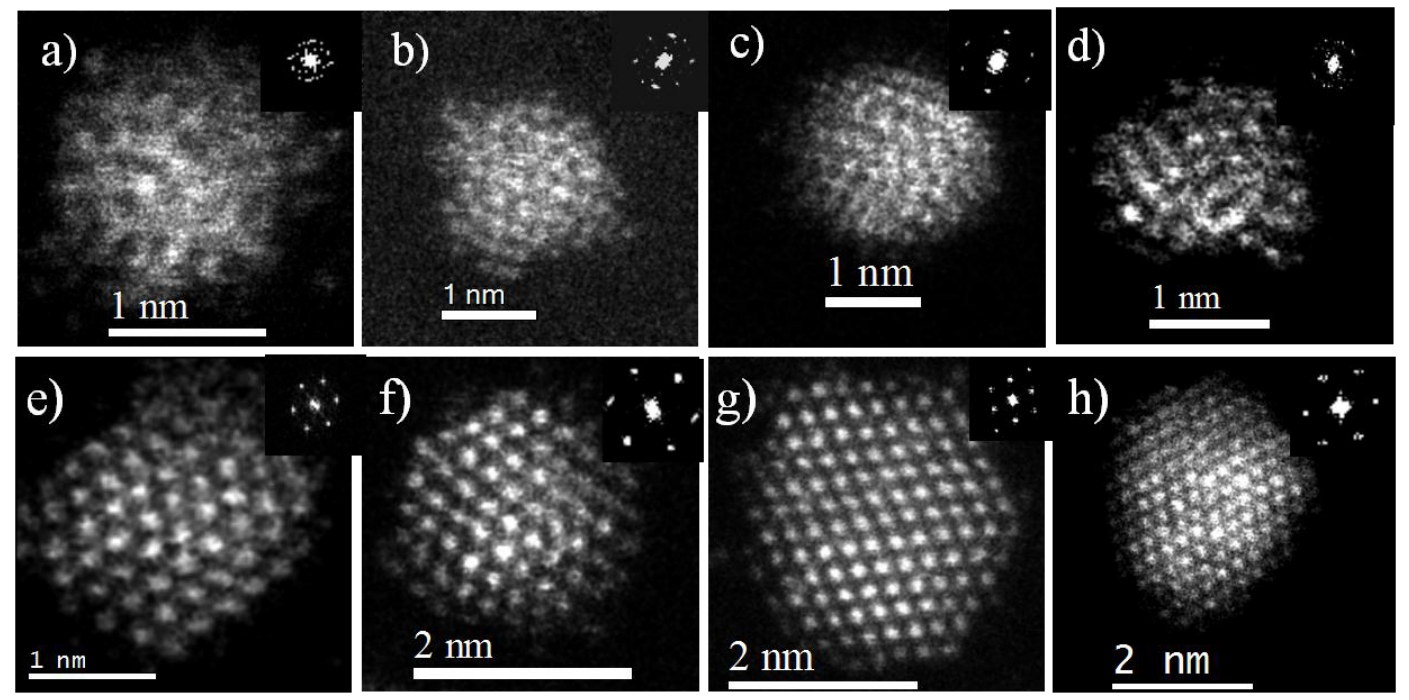

Fig. 2. Some representative HAADF STEM images along different directions. a)-d) $A u_{144}$ nanocluster and e)-h) $\mathrm{Au}_{333}$ nanocluster. The FFT pattern is inset in the corresponding figure.
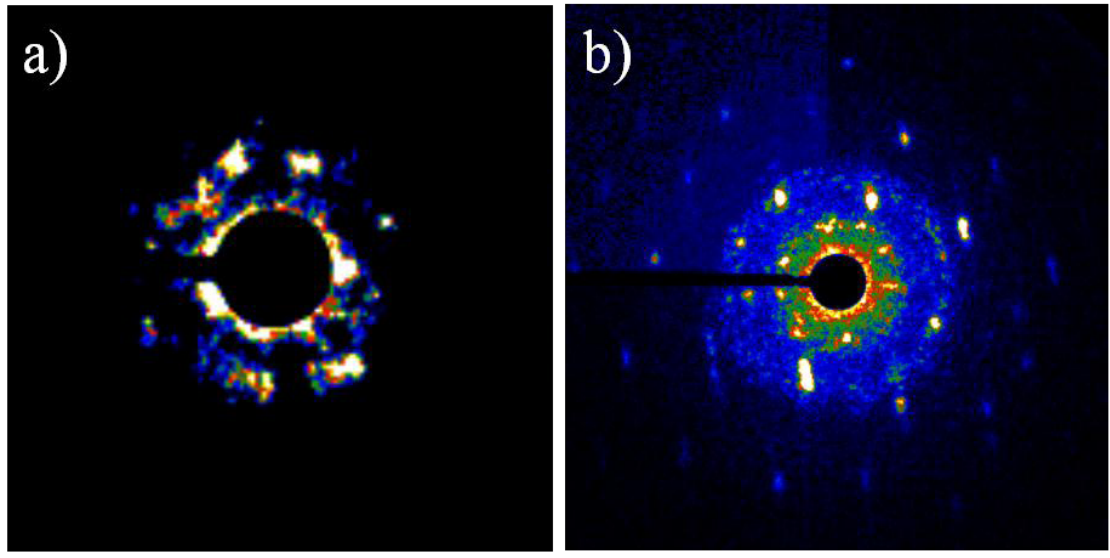

Fig. 3. A representative STEM diffraction patterns from $\mathrm{Au}_{144}$ (a) and $\mathrm{Au}_{333}$ (b) nanocluster. The obtained diffraction pattern is compared with the diffraction patterns from atomistic structural models. 